\title{
Perinatal injuries to the visual cortex enhance the significance of extravisual cortex for performance of a visual habit
}

\author{
HARRY HOWARTH, DONALD R. MEYER, and PATRICIA M. MEYER \\ The Ohio State University, Columbus, Ohio 43210
}

\begin{abstract}
If rats are prepared with visual cortical ablations in early infancy or in adulthood, are trained on a simple visual habit in adulthood, and are finally prepared with second-stage ablations which destroy the rest of the cortex, performance of the habit is markedly affected if, and only if, the injuries to the visual cortex were sustained in infancy. Hence a perinatal visual cortical ablation induces a reorganization of the functions of the extra-visual cortex, and that effect is not producible by injuries to the visual cortex in adulthood.
\end{abstract}

Horel, Bettinger, Royce, and Meyer (1966) examined the effects of second-stage anterior neocortical ablations upon the abilities of rats to remember a black-white discrimination problem that they learned after having been prepared with firststage ablations of the visual neocortex in adulthood. The question was whether the initial injuries would bring about compensatory alterations of the functions of the anterior cortex which could then be detected through comparisons between the animals' performances and those of rats prepared with first-stage anterior ablations. The findings were that animals with first-stage and second-stage ablations of the anterior cortex had similar impairments of retention even though the second-stage subjects had then sustained complete bilateral neocortical ablations.

The same investigators also observed that if rats are prepared with anterior ablations in adulthood and are trained on the problem, the forgetting they exhibit after second-stage ablations of the posterior cortex is neither more nor less severe than the forgetting that is noted when a normal rat is trained on the problem and is tested for retention after having been subjected to a first-stage posterior ablation. The study thus suggested that both subsectors of the dorsolateral cortex, if present, have a role in learning and performance of the problem, but that neither subsector is importantly involved in the process of recovery from an injury to the other subsector.

The work to be described in this report has replicated the first of the foregoing findings. However,

This research was supported by Grant MHO6211 from the National Institute of Mental Health, and was conducted while the senior author was a Fellow in Developmental Psychobiology with support from Training Grant MH15677 from the National Institute of Mental Health. we have also studied the effects of perinatal injuries to the posterior cortex upon the consequences for performance of the problem of second-stage ablations of the anterior cortex in adulthood. We have found that the second-stage ablations then produce effects upon postoperative retention which are no less severe than the effects which are produced by one-stage bilateral ablations of the entire neocortex. The results thus suggest that functions which are normally subserved by the posterior cortex are transferable to other components of the cortex if the posterior cortex is destroyed while the brain is in an early developmental stage.

\section{METHODS}

\section{Subjects and Treatments}

The experiment was carried out with four groups of 22 male Long-Evans hooded rats. The animals of Groups 7P and 63P were subjected to bilateral ablations of the posterior cortex at the ages of 7 days and 63 days, respectively. The animals of Groups 7C and 63C were controls which were subjected to scalp incisions and resuturing at the ages of 7 days and 63 days, respectively. All of the subjects then recovered from their surgeries until they were 98 days old, at which time they were trained to criterion on a black-white discrimination problem. When training was completed, all the subjects were prepared with bilateral ablations of the anterior cortex, were permitted to recover from their second-stage surgeries for a period of 11 days, and thereafter were tested for postoperative retention of performance of the black-white problem.

\section{Surgical Procedures}

The perinatal surgeries for the animals of Groups 7P and 7C were carried out while the subjects were under anesthesias induced with Metafane. In the 7P procedure, the calvarium posterior to the bregma was removed and the underlying dura was incised. The innermost meninges were then gently stripped away with a small cotton swab. The anesthesias for the later operations were induced with sodium pentobarbital; and the ablations were performed through the use of the pia-stripping method in conjunction with aspirations of the tissues at the margins of the lesions (cf. Meyer \& Meyer, 1971).

The lesions of the posterior cortex were intended to include all the regions of the dorsolateral cortex posterior to bregma and be- 
tween the longitudinal fissure and the rhinal fissures. The lesions of the anterior cortex were intended to include the remainder of the dorsolateral cortex. The posterior injuries were sufficiently extensive to include areas $7,17,18,18 \mathrm{a}$, and 20 of Krieg (1946), and hence all the zones of terminations of projections from the dorsolateral geniculate bodies and/or the nuclei lateralis posterior of the thalamus (Hughes, 1977). The anterior injuries occasionally resulted in the transient aphagias which were first observed in anterior subjects by Horel et al. (1966), and which now have been shown by the experiments of Braun (1975) to be produced by injuries to the tongue-taste areas of the anterodorsolateral cortex.

\section{Behavioral Procedures}

The animals were trained in the same apparatus as had been employed by Horel et al. (1966). The rats were required to run from a startbox and then to exit from a choice compartment by knocking down a positive white door that was paired with a negative black door. The incentive was avoidance of shocks to the feet from a grid that formed the floor of the apparatus. The relative positions of the doors were varied in accordance with a Gellermann (1933) series, and 25 training trials were given each day until the subject had reached a criterion of 9 correct responses to the white door in any 10 successive training trials.

The training was preceded by handling and pretraining, the details of which are given by Glendenning (1972). A spaced-trial training procedure was employed in which the animals are rested for 20 min following the 8th and the 16th trials of the 25 daily trials of training. The use of that procedure was the only consequential difference between the behavioral methods of the present study and the study of Horel et al. (1966), who used what we term the massed-trial procedure because there were no rest periods in their daily training sessions. The spaced-trial procedure is the more efficient, but we know of no exception to a rule that a spaced-trial score, if doubled, will predict a comparable massedtrial score (cf. Meyer \& Meyer, 1977).

\section{Histological Procedures}

When retraining was complete, the animals were given a lethal dose of sodium pentobarbital and perfused intracardially with $.9 \%$ saline followed by $10 \%$ Formalin. The brains were removed from the skulls and placed in Formalin for several days. The extents of the cortical ablations were drawn on standard Lashley diagrams, and the brains were then either frozen or embedded in celloidin and serially sectioned at $30 \mu \mathrm{m}$. Every 10th section through the lesions and every fifth section through the thalamus were saved and stained with cresyl violet.

\section{RESULTS}

\section{Behavioral Results}

Figure 1 presents the group-mean numbers of trials which were required for the subjects to learn the problem to successively more stringent criteria of performance prior to their anterior ablations. Group means and standard errors of the means to meet the $9 / 10$ criterion were $39.3 \pm 3.0,30.9 \pm 1.9,33.6 \pm$ 3.6, and $28.6 \pm 2.1$ for Groups $7 \mathrm{P}, 7 \mathrm{C}, 63 \mathrm{P}$, and $63 \mathrm{C}$, respectively. An overall ANOVA yielded an $\mathrm{F}$ ratio of 2.84 ( $\mathrm{df}=3,84)$, which was significant beyond the .05 level. Subsequent $t$ tests showed that the differences between the scores of Group 7P and Groups 7C and 63C were significant beyond the .05 and .01 levels (ts $=2.37,2.94)$. However, more importantly, the scores for Groups $7 \mathrm{P}$ and $63 \mathrm{P}$ were

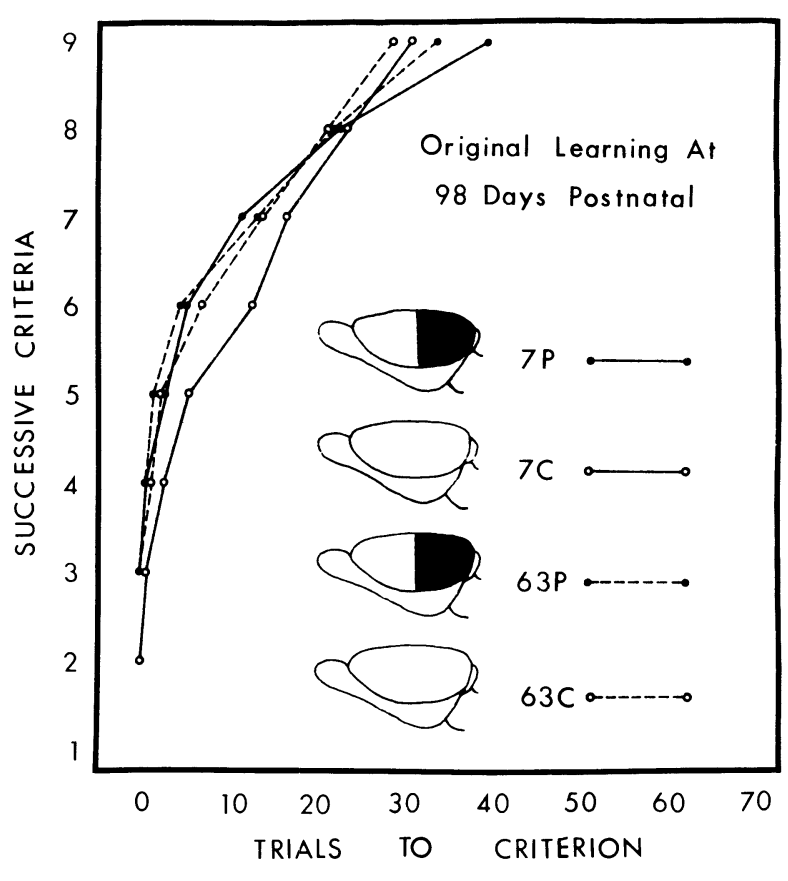

Figure 1. Mean spaced trials required for original learning of the black-white problem to successively more stringent criteria of performance by each of the four groups of subjects. Ns $=22$ per group.

not significantly different $(\mathrm{t}=1.20)$, and the groupmean difference for the latter two groups was only 5.7 trials.

An analysis of errors to criterion was also carried out. The means and standard errors for the errorsto-criterion scores were $18.4 \pm 1.4,17.1 \pm 1.1,15.9 \pm$ 1.6 , and $15.5 \pm 1.1$ for Groups 7P, 7C, 63P, and 63C, respectively. The intergroup differences were not significant as tested by an overall ANOVA, and the difference between the means for Groups 63P and 7P was only 2.5 errors to criterion.

Figure 2 presents the mean trials required for the subjects to relearn the black-white problem following the anterior surgeries. Groups 7C, 63P, and 63C relearned the problem in $19.5 \pm 3.7,16.9 \pm 3.3$, and $17.6 \pm 2.4$ mean trials to the $9 / 10$ criterion, respectively. But Group 7P required $34.5 \pm 5.2$ mean trials to relearn the problem. An overall ANOVA of the trials-to-criterion yielded an $\mathrm{F}$ ratio of 4.78 , significant beyond the .005 level. Subsequent $t$ tests between the mean for Group 7P and the means for Groups $7 \mathrm{C}, 63 \mathrm{P}$, and $63 \mathrm{C}$ were significant beyond the $.05, .01$, and .01 levels, respectively (ts $=2.33$, 2.93, and 2.85). The differences among Groups $7 \mathrm{C}$, $63 \mathrm{P}$, and $63 \mathrm{C}$ failed to reach significance at the .05 levels.

Notably, the difference between the mean trials to the $9 / 10$ criterion for Groups $63 \mathrm{C}$ and $63 \mathrm{P}$ was only 1.1 trials. Moreover, both scores, when doubled as 


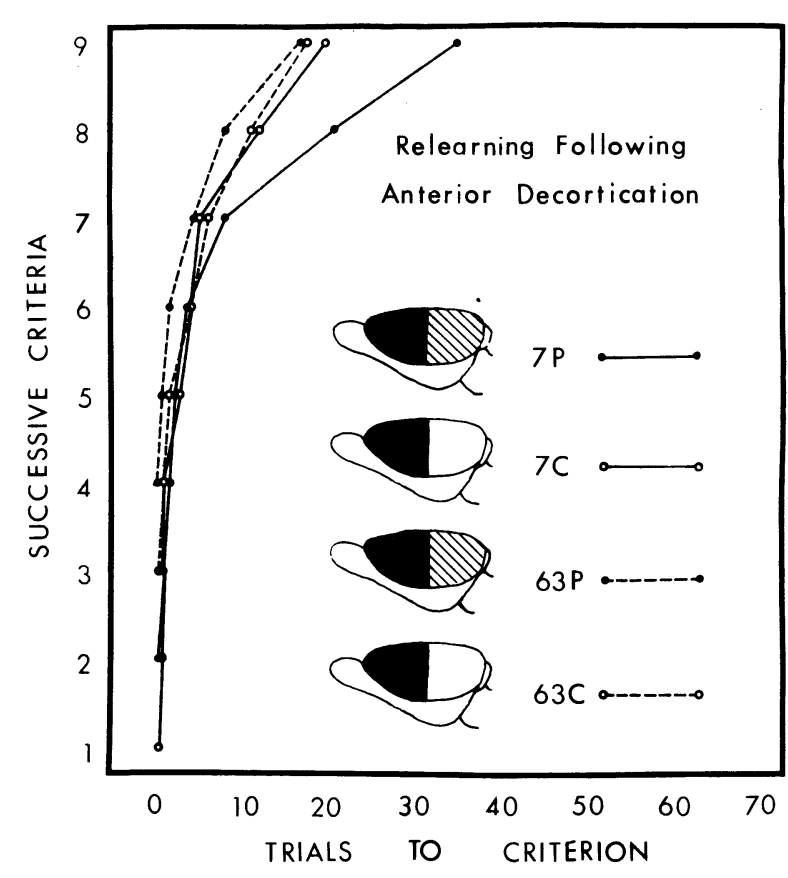

Figure 2. Mean spaced trials required for relearning of the black-white problem to successively more stringent criteria of performance by each of the four groups of subjects. Ns $=22$ per group.

required by Glendenning's (1972) rule for comparisons between spaced-trial and massed-trial scores, corresponded closely to the scores that were obtained by Horel et al. (1966) for relearning of the problem by anterior subjects and by anterior subjects that had also sustained posterior ablations in adulthood prior to original learning of the problem. Specifically, the latter scores were 35.0 and 38.3 mean massed trials, and hence the prediction from the rule was that the subjects of Groups $63 \mathrm{C}$ and $63 \mathrm{P}$ would relearn the problem in approximately 18 trials. The prediction thus derived for Group $63 \mathrm{C}$ was correct to .7 trial, and the prediction thus derived for Group 63P was correct to 1.1 trials.

Relearning of the black-white problem was also measured in terms of errors-to-criterion scores. The means and standard errors of these scores were $17.1 \pm 2.5,11.0 \pm 2.4,8.6 \pm 1.4$, and $9.5 \pm 1.1$ for Groups 7P, 7C, 63P, and 63C, respectively. An overall ANOVA yielded an $F$ ratio of 4.69 , significant beyond the .005 level. Subsequent $t$ test yielded differences significant beyond the .01 levels between Group 7P and Groups 63P and 63C (ts $=3.01$ and 2.81 , respectively), although the differences between Group 7P and Group 7C just failed to reach significance at the .05 level $(t=1.96)$. As was also the circumstance for trials scores, the errors scores for Groups 7C, 63P, and 63C were indistinguishable from each other.
We also examined the relationships between the subjects' individual learning and relearning scores as measured by trials to $9 / 10$ criterion. The Pearson product-moment correlation coefficients were .30 , $-.04, .16$, and -.02 for Groups 7P, 7C, 63P, and 63C, respectively. None of the correlations was statistically significant beyond the .05 level. Accordingly, the findings were similar to those of Jonason, Lauber, Robbins, Meyer, and Meyer (1970) for the relationships between the learning and relearning scores of subjects prepared with posterior injuries in adulthood, and serve once again to question the merits of the use of savings scores in studies of recoveries of functions.

\section{Histological Results}

Figure 3 presents the histological reconstructions of the most and least extensive cortical ablations for each of the four groups. In animals with anterior neocortical surgeries, retrograde degeneration was observed throughout the ventrobasal complex of the thalamus. In animals with posterior lesions, degeneration was observed in NLP and LGNd. When sparing did occur in LGNd, it was confined to the dorsolateral crescent. There were no apparent intergroup differences between the scopes of the ablations for Groups 7P and 63P, or between the scopes of ablations for Groups $7 \mathrm{C}$ and $63 \mathrm{C}$. The subjects within groups were ranked with respect to the extents of their cortical lesions, and Spearman rho correlation coefficients were computed for their rankings and their scores for relearning of the

\section{LESION EXTENT}

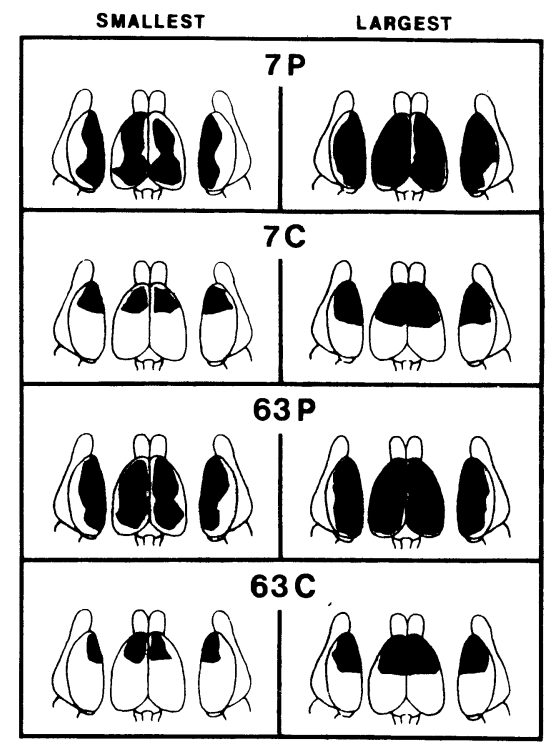

Figure 3. Ranges of extents of the lesions of the cortex for each of the four groups of subjects. 
problem. The coefficients were $-.36,+.13,-.17$, and +.05 for Groups $7 \mathrm{P}, 7 \mathrm{C}, 63 \mathrm{P}$, and $63 \mathrm{C}$, respectively, and none were significant beyond the .05 level.

\section{DISCUSSION}

An injury to the extravisual cortex in adulthood affects a rat's postoperative performance of the black-white problem. The impairment, as was first shown by Horel et al. (1966), is modest and is also completely independent of whether the animal was previously prepared with a first-stage ablation of the posterior cortex in adulthood. However, if the posterior injury is sustained in infancy, and the subject is subsequently trained on the problem in adulthood, the effect of a second stage anterior ablation is almost unbelievably large.

To illustrate, the subjects of Group 7P relearned the problem in 34.5 spaced trials. Hence, the expectation from Glendenning's (1972) rule would be that the 7P subjects would have relearned the problem in approximately 69 massed trials. Meyer, Yutzey, Dalby, and Meyer (1968) observed that rats with onestage ablations of the entire neocortex relearned the problem in 74/64 mean/median massed training trials. Accordingly, the evidence at hand suggests that the costs to the 7P subjects of their secondstage ablations of the anterior cortex were either equal to or very close to the maximal cost of injuries to the cortex for performance of the blackwhite problem.

Importantly, the difference between the performances of Groups 7P and 63P cannot be interpreted as having been a function of differences in recovery times. Thus, the retraining of both groups of subjects began on the 12th day following their second operation, and hence, the only difference between the recovery times for Groups $7 \mathrm{P}$ and 63P was that the subjects of Group 7P had much longer times to recover from their first-stage ablations of the posterior cortex. However, despite that, the 7P subjects were slower to relearn the problem than the 63P subjects, and indeed they required about twice as many trials and committed twice as many errors.
We conclude from the findings of the study as a whole that perinatal injuries to the visual cortex result in alterations of the functions of systems related to the anterior cortex. Their nature is obscure, but they are not producible by posterior injuries in adulthood. The fact that the effect is as large as it is, and is also an effect that depends upon the age of the subject at the time that the visual cortex is destroyed, suggests that the paradigm will be a useful one for studies of the neuroanatomical bases of recoveries from injuries to the brain.

\section{REFERENCES}

Braun, J. J. Neocortex and feeding behavior in the rat. Journal of Comparative and Physiological Psychology, 1975, 89, 507-522.

GellermanN, L. W. Chance orders of alternating stimuli in visual discrimination experiments. Journal of Genetic Psychology, 1933, 42, 207-208.

Glendenning, R. L. Effects of training between two unilateral lesions of visual cortex upon ultimate retention of blackwhite habits by rats. Journal of Comparative and Physiological Psychology, 1972, 80, 216-229.

Horel, J. A., Bettinger, L. A., Royce, G. J., \& Meyer, D. R. Role of neocortex in the learning and relearning of two visual habits by the rat. Journal of Comparative and Physiological Psychology, 1966, 61, 66-78.

Hughes, H. C. Anatomical and neurobehavioral investigations concerning the thalamo-cortical organization of the rat's visual system. Journal of Comparative Neurology, 1977, 175, 311-335.

Jonason, K. R., Lauber, S. M., Robbins, M. J., Meyer, P. M., \& MEYER, D. R. Effects of amphetamine upon relearning pattern and black-white discriminations following neocortical lesions in rats. Journal of Comparative and Physiological Psychology, 1970, 73, 47-55.

KrIEG, W. J. S. Connections of the cerebral cortex. I. The albino rat. A. Topography of the cortical areas. Journal of Comparative Neurology, 1946, 84, 221-275.

Meyer, P. M., \& MEYer, D. R. Neurosurgical procedures with special reference to aspiration lesions. In R. B. Myers (Eds), Methods in neuro-biology. New York: Academic Press, 1971.

Meyer, P. M., Yutzey, D. A., Dalby, D. A., \& Meyer, D. R. Effects of simultaneous septal-visual, septal-anterior and anterior-posterior lesions upon relearning a black-white discrimination. Brain Research, 1968, 8, 281-290.

(Received for publication January 4, 1979; revision accepted April 20, 1979.) 